\title{
Porous Para-aminobenzoic Acid Membrane Prepared by Electrochemical Method and Its Application in Voltammetric Determination of Dopamine
}

\author{
Qiong WU ${ }^{\mathrm{a}}$, Hanshuang ZHANG ${ }^{\mathrm{b}}$, Meixia $\mathrm{WANG}^{\mathrm{c}}$ and Huaixiang $\mathrm{LI}^{\mathrm{d}^{*}}$ \\ College of Chemistry, Chemical Engineering and Materials Science, Collaborative Innovation Center \\ of Functionalized Probes for Chemical Imaging in Universities of Shandong, Key Laboratory of \\ Molecular and Nano Probes, Ministry of Education, Shandong Provincial Key Laboratory of Clean \\ Production of Fine Chemicals, Shandong Normal University, Jinan 250014, P. R. China \\ a785202295@qq.com¹, ${ }^{\mathrm{b}} 756322383 @$ qq.com, , ${ }^{\mathrm{c}} 2542225637 @ q q . c o m,{ }^{d}$ lihuaixiang@sdnu.edu.cn
}

\begin{abstract}
In this paper, we prepared poly para aminobenzoic acid (PABA) membrane on a glassy carbon electrode (GCE) using cyclic voltammetry $(\mathrm{CV})$ following electrochemically degrading to form a porous PABA (PPABA) membrane. The PPABA membrane was characterized using CV technique, scanning electron microscope (SEM) measurements. The PPABA membrane could improve sensitivity of dopamine (DA) and inhibit ascorbic acid (AA) and uric acid (UA) electrochemical oxidation. The PPABA/GCE-based electrohemical sensor displayed a good linear ranges from $0 \mu \mathrm{M}$ to $30 \mu \mathrm{M}$ DA with a low detection limits of $1.6 \mu \mathrm{M}$ DA $(\mathrm{S} / \mathrm{N}=3)$ in voltammetry measurement. The proposed sensor has the advantages of being simple, reproducible and stable and can be used as a new sensitive electrochemical biosensor for the detection of DA.
\end{abstract}

\section{Introduction}

Nowadays, polymer membranes are becoming more and more compelling due to their growing demand in environment, energy and health fields. Many efforts have been devoted to improve the membrane performance and extend their application via the methodology of surface engineering. One of the most promising strategies is mussel-inspired chemistry, which has become a powerful tool in membrane fabrication and modification because of its universality and versatility [1]. It is reported that para-aminobenzoic acid (p-ABA) could be easily electropolymerized on various substrate materials to form poly-para-aminobenzoic acid (PABA) films with good chemical and mechanical stability [2]. Since the amino group is an important functional group in PABA that undergoes oxidation on the electrode surface, the modification of the electrode surface is supposed to increase the sensitivity of it [3]. However, electrochemical synthesis of PABA membranes followed by electrochemical degradation to produce porous poly-para-aminobenzoic acid (PPABA) film has not been

*Corresponding author: 785202295@qq.com 
reported yet as far as we know. This basic information is useful and important for us to explore the fundamental characteristics of PABA polymer membranes and to further optimize their performances for diverse potential applications in water treatment, bioseparation and blood purification [4].

Dopamine (DA) plays a significant role in the function of the central nervous, renal, hormonal and cardiovascular system [5]. It is important to monitor DA sensitively and selectively not only for biomedical chemistry and neurochemistry research but also for diagnostic and pathological purpose [6]. Thus, great attention has been paid to the development of functional electrode systems for detection of DA. It is well known that the selective detection of DA in biological media requires differentiation from the signal interference due to ascorbic acid (AA), which exhibits an oxidation potential close to that of DA and results in voltammetric response overlap [7]. In addition, a considerably higher concentration of AA than DA in real samples makes selective detection of DA even more difficult. To solve these problems, chemically modified electrodes were applied in the selective analysis of DA. Various materials can be utilized in the fabrication of modified electrode due to their excellent properties to decrease the over-potential, accelerate the electron and mass transfer rate or enrich the substrates on the electrode surfaces [8]. Construction of DA electrochemical sensors based on the PABA degradation by electrochemical method has not yet been reported. According to the principle of PABA degradation by cyclic voltametry $(\mathrm{CV})$, this study reports a new electrochemical sensor for DA based on the electrically polymerized PABA membrane and then gradually degraded by electrochemical method and cause the changes of membrane interface structure and the electrochemical signal, thus realizing the selective detection of DA. The porous membranes were characterized by $\mathrm{CV}$, scanning electron microscope (SEM) measurement.

\section{Experimental Method}

Materials and Apparatus. Para-aminobenzoic acid (p-ABA), potassium ferricyanide, $\mathrm{Na}_{2} \mathrm{HPO}_{4} \cdot 12 \mathrm{H}_{2} \mathrm{O}, \mathrm{NaH}_{2} \mathrm{PO}_{4} \cdot 2 \mathrm{H}_{2} \mathrm{O}$, dopamine (DA) hydrochloride, and other reagents were obtained from Sinopharm Chemical Reagent Co. Ltd. (Shanghai, China). All other reagents were of analytical reagent grade. All solutions were prepared with dissolved in doubly distilled water. A phosphate buffer solution (PBS) was made of $0.1 \mathrm{M} \mathrm{Na}_{2} \mathrm{HPO}_{4}$ and $0.1 \mathrm{M}$ $\mathrm{NaH}_{2} \mathrm{PO}_{4}$ and adjusted to $\mathrm{pH}=7$ either using $\mathrm{KOH}(0.1 \mathrm{M})$ or $\mathrm{HCl}(0.1 \mathrm{M})$. All cyclic voltametry $(\mathrm{CV})$ experiments were performed on a LK2005 electrochemical workstation (Lanlike Company, Tianjin, China). A conventional three-electrode system was applied. The working, the auxiliary, and the reference electrodes were modified glassy carbon electrode (GCE, diameter: $3 \mathrm{~mm}$ ), platinum plate, and saturated calomel electrode (SCE), respectively.

Electropolymerization of Membrane. Prior to experiment, a GCE with the diameter of $3.0 \mathrm{~mm}$ was polished with diamond paste and ultrasonic cleaned sequentially in acetone, $\mathrm{KOH}(1.0 \mathrm{M}), \mathrm{HNO}_{3}(1: 1, \mathrm{v} / \mathrm{v})$ and doubly distilled water. Poly-para-aminobenzoic acid (PABA) membranes were prepared by cycling the electrode potential between -0.60 and $+1.00 \mathrm{~V}$ (vs. SCE), $25 \mathrm{CV}$ scans at the scan rate of $50 \mathrm{mV} \cdot \mathrm{s}^{-1}$ in $0.1 \mathrm{M}$ PBS containing $6 \mathrm{mM}$ p-ABA.

Membrane Degradation by $\mathbf{C V}$ in KOH Solution. The electrosynthesized PABA films were electrochemically degraded by cycling the potential between -0.80 and $+0.80 \mathrm{~V}$ (vs. SCE), $15 \mathrm{CV}$ scans in $0.05 \mathrm{M} \mathrm{KOH}$ solution at the scan rate of $80 \mathrm{mV} \cdot \mathrm{s}^{-1}$ to produce porous PABA membrane (PPABA) on the GCE.

Characterization of the Membrane. Electrochemical characterizations were also made by the CV technique. A CV cycle was realized between -0.80 and $0.80 \mathrm{~V}$ (vs. SCE), at a scan rate of $20 \mathrm{mV} \cdot \mathrm{s}^{-1}$. For these experiments, the electrolyte is a solution of potassium hexacyanoferrate trihydrate at $3 \mathrm{mM}$ (Sigma Aldrich) containing potassium chloride $(0.10$ 
M). A glassy carbon plate electrode of surface area $1.0 \mathrm{~cm}^{2}$ was utilized for electrodeposition/electrodegradation and used for scanning electron microscope (SEM). TA FEI Sirion 200 SEM (Netherlands) was used to observe the surface morphology of the modified membranes.

Electrochemical Measurements for Selective Detection DA. Standard solution of DA and AA were prepared using doubly distilled water as solvent and stored under $4{ }^{\circ} \mathrm{C}$. Electrochemical analysis of DA on the modified electrodes was carried out in $0.10 \mathrm{M}$ PBS containing a certain amount of DA. The CV cycles were realized between -0.6 and $0.6 \mathrm{~V}$ (vs. $\mathrm{SCE}$ ), at a scan rate of $50 \mathrm{mV} \cdot \mathrm{s}^{-1}$. After each measurement, the modified electrode was polished and washed with water and scanned in blank PBS until the electrochemical signals of DA were disappeared.

\section{Results and Discussion}

Electropolymerization and Electrochemical Degradation of PABA Membranes. CV is a powerful technique to carry out electrochemical reactions at the working electrode's surface. It was thus chosen to fabricate membranes. PABA polymerization was performed directly onto the GCE surface, which greatly simplified the fabrication procedure.

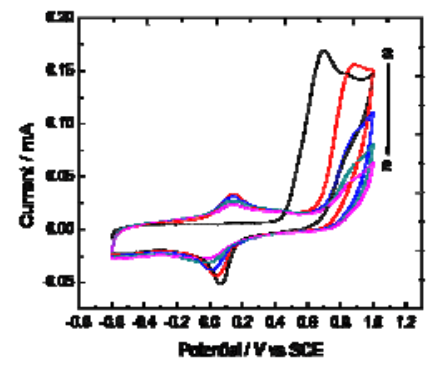

Fig. 1. Cyclic voltammograms for the electropolymerization of $3 \mathrm{mM} \mathrm{p}-\mathrm{ABA}$ in a $0.1 \mathrm{M}$ PBS (pH 7.0) on a GCE. Scan rate: $50 \mathrm{mV} \mathrm{s}^{-1}$; number of scan cycles: 25 . Number of cycles: a 1, b 4, c 7, d 12, e 25 .

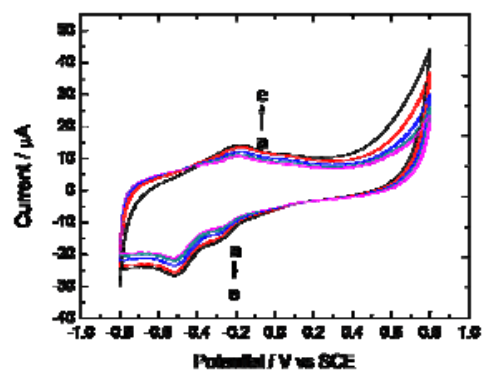

Fig. 2. Cyclic voltammograms for the electrochemical degradation of PABA $0.05 \mathrm{M}$ KOH solution on the GCE. Scan rate: $80 \mathrm{mV} \mathrm{s}^{-1}$; number of scan cycles: 15 . Number of cycles: a 1,b 2, c 5, d 10, e 15.

Fig. 1 shows the cyclic voltammograms recorded during the electropolymerization of 6 $\mathrm{mM}$ p-ABA on the GCE at a scan rate of $50 \mathrm{mV} \cdot \mathrm{s}^{-1}, 25$ scan cycles. When the potential 
ranged from -0.60 to $1.00 \mathrm{~V}$, an irreversible oxidation peak appeared at $0.67 \mathrm{~V}$, which could be attributed to the formation of an amino cation radical [9]. This oxidation peak current reduced rapidly and disappeared when the completion of the seven cycles. Of course, a pair of oxidation and reduction peaks was observed at about 0.11 and $0.08 \mathrm{~V}$, which may be ascribed to the oxidation and reduction of the PABA film, and the peak current intensities of the redox pair became smaller with the number of scans increased until reaching a certain limit, which is in agreement with the results reported by other authors [10], which shows a continuous growth of the poor conductive layer on the surface of the GCE. These observations indicate that an adherent PABA film was electrodeposited on the surface of the GCE.

Fig. 2 shows the degradation process of the PABA in a solution of potassium hydroxide. The degradation was performed by 15 cycles the potential between -0.80 and $0.80 \mathrm{~V}$ (vs. $\mathrm{SCE}$ ) in $0.05 \mathrm{M} \mathrm{KOH}$ solution. As the increase of scans, the oxidation peak at about $-0.20 \mathrm{~V}$ and reduction peak at about $-0.55 \mathrm{~V}$ decreased gradually, could be ascribed degradation of PABA.

Characterization of PABA Membranes. Fig. 3 presents the characterizations of cyclic voltammograms with the $\mathrm{Fe}(\mathrm{CN})_{6}{ }^{3-} / \mathrm{Fe}(\mathrm{CN})_{6}{ }^{4-}$ redox activity at a bare $\mathrm{GCE}$ (a), as-prepared PABA modified GCE (b) and after electrochemically degraded PPABA/GCE (c). A couple of typical redox peaks of hexacyanoferrate appears on the bare GCE (curve a). The as-prepared PABA membrane modified GCE shows a compact layer at the surface of the working electrode, impeding the access of the redox couple of ferricyanide/ferrocyanide to the electrode (curve b). However, as shown curve (c) in Fig.3, an obvious current increase appears when using the degraded PPABA/GCE under same experimental conditions. Although the modified layer insulates the GCE to a large extent, the redox peak current can still be seen in the voltammogram. This may result from the cavities caused by the electrochemical degradation of PABA to form some channels for hexacyanoferrate to arrive at the electrode surface. Certainly, the peak currents clearly are smaller than those at the bare GCE. These results are accordant with scanning electronic microscope (SEM) observation as described in Fig. 4 as follows. Fig. 4 shows SEM images of as-formed PABA (A) and electrochemical degraded PPABA (B). We could see from the SEM images that the surface of GCE modified with PABA membrane is compact and random sphere, but after electrochemical degradation, some cavities and channels can be observed from the electrode surface.

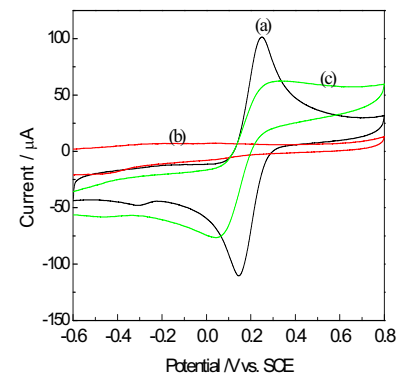

Fig. 3. CVs of $5 \mathrm{mM} \mathrm{K}{ }_{3} \mathrm{Fe}(\mathrm{CN})_{6} / \mathrm{K}_{4} \mathrm{Fe}(\mathrm{CN})_{6}$ in $0.1 \mathrm{M} \mathrm{KCl}$ solution, (a) bare $\mathrm{GCE}$, (b) as prepares PABA and (c) after electrochemical degradation of PPABA. 


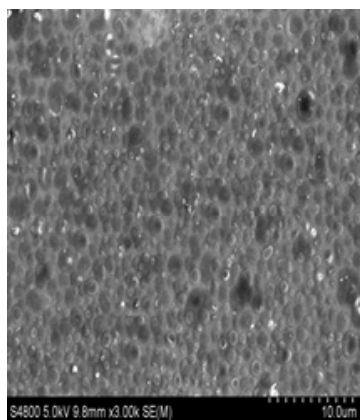

(A)

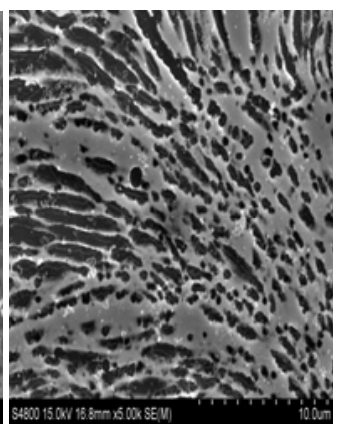

(B)

Fig. 4. (A) SEM image of as prepared poly para-aminobenzoic acid modified on GCE. (B) SEM image of PPM modified GCE after electrochemical degradation.

In brief, the redox peaks of hexacyanoferrate increase in CV and the SEM images both indicate that a porous poly para-aminobenzoic acid membrane can be fabricated by electrochemical degradation of a poly para-aminobenzoic acid membrane on the GCE.

Electrochemical Determination of DA. Because of the strong adsorption property of PPABA for DA, it was intended to develop a DA assay using PPABA/GCE.
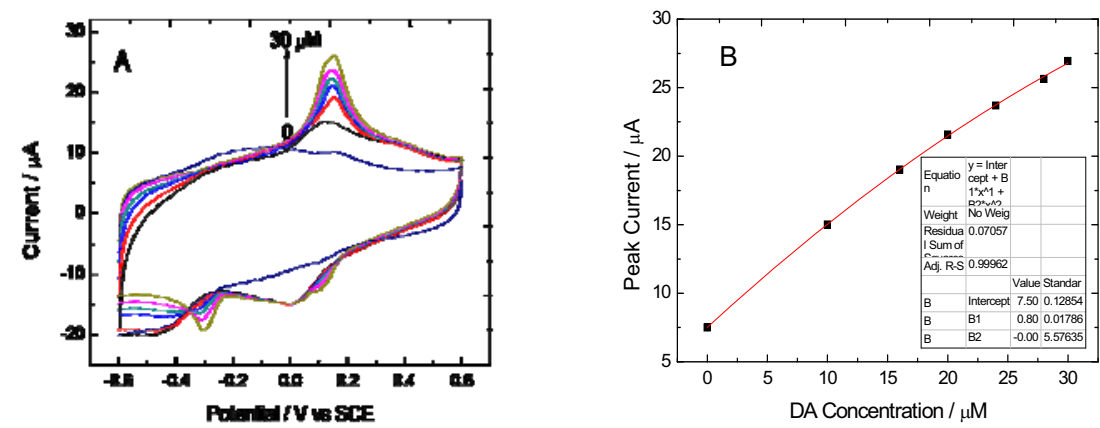

Fig. 5. (A) Cyclic voltammograms of PPABA/GCE $0.1 \mathrm{M} \mathrm{pH} 7 \mathrm{PBS}$ with different concentration of (a) 0 , (b) 10, (c) 16, (d)20, (e) 24, (f) 28 and (g) $30 \mu \mathrm{M}$ DA at scan rate $50 \mathrm{mV} \cdot \mathrm{s}^{-1}$. (B) Plots of peak current vs. DA concentration using the data depicted in part A.

Fig. 5(A) shows the well-defined CV oxidation peaks under optimized conditions, and the currents increased with the increasing concentration of the DA standard solutions. Fig. 5B shows the linear calibration curve from 0 to $30 \mu \mathrm{M}$ with slope and regression coefficient of $0.617 \mu \mathrm{A} / \mu \mathrm{M}$ and 0.996 , respectively. The detection limit is estimated to be $1.6 \mu \mathrm{M}$, defined as three times the standard deviation of the blank measurement in the absence of target analyte divided by the slope of the calibration plot. This lower detection limit and higher sensitivities are essential to ensure the accuracy of measurements in applications. In addition, a simple and controllable preparation and degradation of composite polymer membrane on GCE would provide efficient detection of DA from the technological point of view.

The electrochemical performance of two important anionic interferences, AA and UA, using the proposed electrodes were further evaluated. From cyclic voltammograms no obvious peaks were observed for $20,40,100 \mu \mathrm{M}$ AA or $40,100 \mu \mathrm{M}$ UA at the PPABA/GCE. 
The results show that the electrochemical activity of AA and UA is very weak at the modified electrode.

\section{Summary}

A porous poly para-aminobenzoic acid membrane was formed by electrically polymerizing para-aminobenzoic acid and then gradually electrochemically degrading poly para-aminobenzoic acid. A new type of electrochemical sensor for determination of DA based on porous poly para-aminobenzoic acid membrane modified glassy carbon electrode has been proposed. Results show that porous poly para-aminobenzoic acid membrane can inhibit both AA and UA electrochemical activity. The optimized sensor displayed excellent linear response in $\mathrm{CV}\left(\mathrm{R}^{2}=0.996\right)$ measurements. A low detection limit $(1.6 \mu \mathrm{M}, \mathrm{S} / \mathrm{N}=3)$ toward DA was obtained in CV measurement. Moreover, the structure of the porous poly para-aminobenzoic acid membrane maintains a long stability for measurements and storage.

\section{Acknowledgements}

This work was supported by the National Natural Science Foundation of China (No: 21405096) and University Student Innovation Training Project of China (No: 20151504).

\section{References}

1. V. Ball, D.D. Frari, M. Michel, M.J. Buehler, V. Toniazzo, M.K. Singh, J. Gracio, D. Ruch, Deposition mechanism and properties of thin polydopamine films for high added value applications in surface science at the nanoscale, BioNanoSci. 2 (2012) 16-34.

2. Y.T. Liu, J. Deng, X.L. Xiao, L. Ding, Y.L. Yuan, H. Li, X.T. Li, X.N. Yan, L.L. Wang, Electrochemical sensor based on a poly(para-aminobenzoic acid) film modifiedvglassy carbon electrode for the determination of melamine in milk, Electrochim. Acta 56 (2011) 4595-4602.

3. L. Shang, F. Zhao, B. Zeng, 3D porous graphene-porous $\mathrm{PdCu}$ alloy nanoparticles-molecularly imprinted poly(para-aminobenzoic acid) composite for the electrocatalytic assay of melamine, ACS Appl. Mater. Interfaces 6 (2014) 18721-18727.

4. J.H. Jiang, L.P. Zhu, H.T. Zhang, B.K. Zhu, Y.Y. Xu, Improved hydrodynamic permeability and antifouling properties of poly(vinylidene fluoride) membranes using polydopamine nanoparticles as additives, J. Membr. Sci. 457 (2014) 73-81.

5. Y. Wang, Y. Li, L. Tang, J. Lu, J. Li, Application of graphene-modified electrode for selective detection of dopamine, Electrochem. Commun. 11 (2009) 889-892.

6. C. Wang, J. Du, H. Wang, C. Zou, F. Jiang, P. Yang, Y. Du, A facile electrochemical sensor based on reduced graphene oxide and Au nanoplates modified glassy carbon electrode for simultaneousdetection of ascorbic acid, dopamine and uric acid, Sens. Actuators B. 204 (2014) 302-309.

7. Z. Jia, J. Liu, Y. Shen, Fabrication of a template-synthesized gold nanorod-modified electrode for the detection of dopamine in the presence of ascorbic acid, Electrochem. Commun. 9 (2007) 2739-2743.

8. S. Elhag, Z.H. Ibupoto, X. Liu, O. Nur, M. Willander, Dopamine wide range detection sensor based on modified $\mathrm{Co}_{3} \mathrm{O}_{4}$ nanowires electrode, Sens. Actuators B 203 (2014) 543-549.

9. Y.T. Liu, J. Deng, X.L. Xiao, L. Ding, Y.L. Yuan, H. Li, X.T. Li, X.N. Yan, L.L. Wang, Electrochemical sensor based on a poly(para-aminobenzoic acid) film modifiedvglassy 
carbon electrode for the determination of melamine in milk, Electrochim. Acta 56 (2011) 4595-4602.

10. R.M. Kotkar, A.K. Srivastava, Voltammetric determination of para-aminobenzoic acid using carbon paste electrode modified with macrocyclic compounds, Sens. Actuators B. 119 (2006) 524-530. 